\title{
Electron/positron measurements obtained with the Mars Science Laboratory Radiation Assessment Detector on the surface of Mars
}

\author{
J. Köhler ${ }^{1}$, R. F. Wimmer-Schweingruber ${ }^{1}$, J. Appel ${ }^{1}$, B. Ehresmann ${ }^{2}$, C. Zeitlin ${ }^{6, a}$, D. M. Hassler ${ }^{2}$, G. Reitz ${ }^{3}$, \\ D. E. Brinza ${ }^{5}$, S. Böttcher ${ }^{1}$, E. Böhm ${ }^{1}$, S. Burmeister ${ }^{1}$, J. Guo ${ }^{1}$, A.-M. Harri ${ }^{7}$, H. Kahanpää ${ }^{7,8}$, J. Krauss ${ }^{1}$, H. Lohf ${ }^{1}$, \\ C. Martin ${ }^{1}$, D. Matthiä ${ }^{3}$, A. Posner ${ }^{4}$, and S. Rafkin ${ }^{2}$ \\ ${ }^{1}$ Institute of Experimental and Applied Physics, Christian-Albrechts-University, Kiel, Germany \\ ${ }^{2}$ Southwest Research Institute, Space Science and Engineering Division, Boulder, CO, USA \\ ${ }^{3}$ Institute of Aerospace Medicine, German Aerospace Center (DLR), Cologne, Germany \\ ${ }^{4}$ NASA Headquarters, Science Mission Directorate, Washington DC, USA \\ ${ }^{5}$ Jet Propulsion Laboratory, California Institute of Technology, Pasadena, CA, USA \\ ${ }^{6}$ Southwest Research Institute, Earth, Oceans \& Space Department, Durham, NH, USA \\ ${ }^{7}$ Finnish Meteorological Institute, Helsinki, Finland \\ ${ }^{8}$ Aalto University, Espoo, Finland \\ anow at: Lockheed Martin Information Systems \& Global Solutions, Houston, TX, USA
}

Correspondence to: J. Köhler (koehler@physik.uni-kiel.de)

Received: 1 September 2015 - Revised: 13 November 2015 - Accepted: 12 January 2016 - Published: 27 January 2016

\begin{abstract}
The Radiation Assessment Detector (RAD), on board the Mars Science Laboratory (MSL) rover Curiosity, measures the energetic charged and neutral particles and the radiation dose rate on the surface of Mars. Although charged and neutral particle spectra have been investigated in detail, the electron and positron spectra have not been investigated yet. The reason for that is that they are difficult to separate from each other and because of the technical challenges involved in extracting energy spectra from the raw data. We use GEANT4 to model the behavior of the RAD instrument for electron/positron measurements. We compare Planetocosmics predictions for different atmospheric pressures and different modulation parameters $\Phi$ with the obtained RAD electron/positron measurements. We find that the RAD electron/positron measurements agree well with the spectra predicted by Planetocosmics. Both RAD measurements and Planetocosmics simulation show a dependence of the electron/positron fluxes on both atmospheric pressure and solar modulation potential.
\end{abstract}

Keywords. Interplanetary physics (cosmic rays; energetic particles; instruments and techniques)

\section{Introduction}

The Mars Science Laboratory (MSL) Curiosity rover has been operating in Gale crater on Mars since its landing on 6 August 2012. The Radiation Assessment Detector (RAD) on board provides the first ever radiation measurements on the surface of Mars. With the exception of some short interruptions, RAD provides a continuous radiation measurement from the Martian surface, operating in a 16, 32 or 58 min observation cadence. Two of the primary science objectives of RAD are "to measure energetic particle spectra at the surface of Mars" and "to use these measurements to enable validation of Mars atmospheric transmission models and radiation transport codes" (Hassler et al., 2012). These objectives are directly related and of prime importance to assessing possible constraints for future human exploration of Mars.

In Köhler et al. (2014) gamma and neutron spectra have been calculated from RAD data and were compared to Planetocosmics simulations. In Ehresmann et al. (2014) the energy spectra of protons and heavier elements have been calculated from RAD data and were compared to OLTARIS simulations.

In contrast to other charged particles, electrons and positrons create ambiguous signals in RAD that are chal- 
lenging to interpret. Therefore, we use the Planetocosmics transport code to model the expected electron and positron energy spectra on the Martian surface and then use a detailed GEANT4 model of the RAD instrument to simulate the measurement process. These modeled results are compared with RAD data.

\subsection{The Martian radiation environment}

The Martian radiation environment is mainly generated by galactic cosmic rays (GCR). GCRs consist of $98 \%$ atomic nuclei and $2 \%$ electrons, the nuclei can be further divided into $\sim 90 \%$ protons, $9 \%$ helium, and $1 \%$ heavier nuclei (Simpson, 1983). The radiation environment on the Martian surface consists of primary GCRs, which penetrate the thin Martian atmosphere (19-23 $\mathrm{g} \mathrm{cm}^{-2}$ at Gale crater), and of secondary particles produced by the interaction of GCRs with atmosphere and soil. This radiation environment has been modeled in several existing studies (Ehresmann et al., 2011; Dartnell et al., 2007a, b; Keating et al., 2005; McKenna-Lawlor et al., 2012), and measured with the RAD instrument (Ehresmann et al., 2014; Köhler et al., 2014; Hassler et al., 2014).

The GCR-induced Martian radiation environment is influenced by several periodic effects on different timescales. The GCR flux is modulated by the solar magnetic field which correlates with the 11-year solar activity cycle and the 27-day solar rotation. Seasonal and diurnal pressure changes in the Martian atmosphere also affect the radiation environment on the Martian surface. These influences on the surface radiation field haven been observed by RAD (Rafkin et al., 2014; Guo et al., 2015). Primary GCRs going through the Martian atmosphere generate secondaries and can even cause an increase of the flux above a certain height - the Pfotzer Maximum which is located $\sim 5$ to $10 \mathrm{~cm}$ below the surface of Mars. This means that with increased atmospheric column density, we would expect increased fluxes, especially for secondaries. However the RAD measured surface dose rate is inversely related to the surface pressure (Rafkin et al., 2014; Guo et al., 2015). This is because dose rate is a combination of the total deposited energy by all detected particles where the main contribution comes from high-charge and energy primary particles (so-called HZE particles) on which the atmosphere has a shielding effect due to nuclear fragmentation. Secondary particles on the other hand, are not only influenced by the atmosphere's shielding effect but also by the atmosphere's generating effect. The secondary particles on the Martian surface should therefore increase with atmospheric column density as seen, e.g., in the neutral particle count rate presented in Rafkin et al. (2014). The fluxes of electrons and positrons at the Martian surface are mainly secondaries, therefore, their fluxes are expected to increase with atmospheric column density.

In addition to the GCR-induced radiation environment, solar particle events can cause intermittent enhancements in the observed particle radiation on short timescales (Cleghorn et al., 2004; Hassler et al., 2014). In the time frame considered in this work (August 2012 to May 2015) only four Solar Energetic Particle (SEP) events were directly observed by RAD on the surface.

In this work we model the Martian radiation environment with Planetocosmics (Desorgher et al., 2006) and compare the obtained results to RAD electron/positron measurements.

\subsection{The RAD instrument}

The RAD instrument, shown schematically in Fig. 1, houses several detectors for the measurement of energetic charged and neutral particles. The silicon detectors (A, B, C) form a telescope which is followed by a CsI scintillator (D) that preserves the telescope's viewing cone, a plastic scintillator (E), and an additional plastic scintillator (F) that surrounds $\mathrm{D}$ and $\mathrm{E}$ and acts as anti-coincidence for neutral particle detection. The silicon detectors are segmented into inner and annular, outer segments. For charged particle measurements, only the inner segments of $\mathrm{B}$ and $\mathrm{C}$ are used. Together with the inner (A2) and outer (A1) segment of A, B and C inner segments form two different field of view cones. Stopping charged particles requires a coincidence between $\mathrm{A}$ and $\mathrm{B}$ and an anti-coincidence with $\mathrm{E}$ and $\mathrm{F}$. A detailed description of the charged particle measurement is given in Ehresmann et al. (2014) and a complete description of the instrument is given in Hassler et al. (2012).

In Ehresmann et al. (2014) energy and species of stopping charged particles (ranging from protons to iron) are identified in a so-called Goulding plot. In contrast to those comparatively heavy particles, energy and species of electrons and positrons can not be identified unambiguously in such a Goulding plot. Therefore, we use the GEANT4 toolkit (Agostinelli et al., 2003) to model and understand electron/positron measurements and to compare Planetocosmics predictions with RAD measurements from the surface of Mars.

\section{Method}

\subsection{Planetocosmics simulations}

To obtain the expected electron and positron fluxes on the Martian surface, we use a Planetocosmics version based on GEANT 4.9.6. As a setup of the Martian atmospheric environment, detailed pressure profile and composition were selected based on the Mars climate database 5.0 (Lewis et al., 1999).

As input for the Planetocosmics simulation we use the proton and helium GCR spectra as given by the BadhwarO'Neill 2010 Galactic Cosmic Ray Flux Model (O'Neill, 2010) and the electron/positron spectra GCR spectra as given in Potgieter and Nndanganeni (2013); Adriani et al. (2011). Since previous work has shown that the cosmic modulation, 


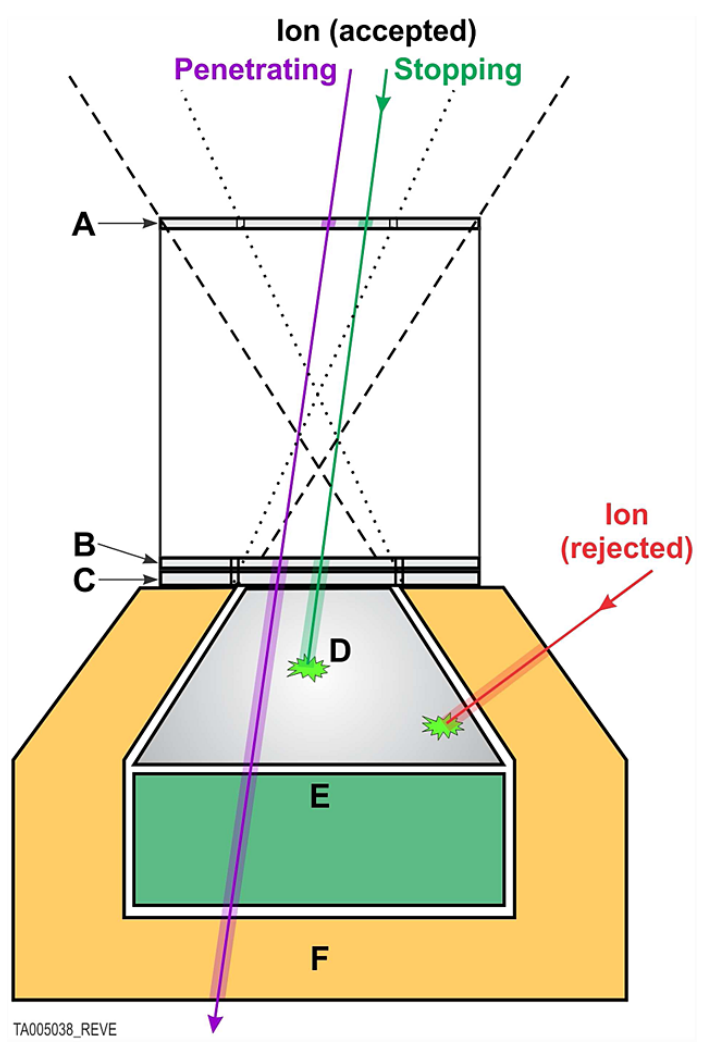

Figure 1. Schematic of the RAD instrument. The detectors A, B, C, $\mathrm{D}$ form a telescope for the measurement of charged particles from above. A is segmented in an inner (A2) and an outer (A1) segment, which form two field of view cones, shown by the dashed and dotted lines respectively. Stopping charged particles requires a coincidence between $\mathrm{A} 2$ or $\mathrm{A} 1$ and $\mathrm{B}$, and an anti-coincidence with $\mathrm{E}$ and $\mathrm{F}$. The green arrow illustrates an example of a stopping charged particle while the red and magenta arrows stand for particles with trajectories that make them unsuitable for the present analysis. Figure is taken from Ehresmann et al. (2014).

as well as atmospheric pressure has a clear influence on the RAD dose rate measurements (Guo et al., 2015), we obtain electron/positron spectra for a range of solar modulation parameters and Martian atmospheric pressures which are typical values measured during the same time period. The cosmic ray modulation parameter $\Phi$ (a measure of deceleration of particles as they traverse the heliosphere to reach $1 \mathrm{AU}$ ) is set to be 300,600 , and $900 \mathrm{MV}$, and we use atmospheric pressures of $748 \mathrm{~Pa}$ (minimum annual pressure) and $886 \mathrm{~Pa}$ (maximum annual pressure).

Figure 2 shows the resulting spectra. As expected from (Guo et al., 2015), the electron and positron fluxes increase with pressure and decrease with increasing $\Phi$. Muons of either charge may by falsely identified as electrons or positrons, and therefore contaminate the measurement. Consequently, their fluxes haven been calculated and shown in the figure as well. Below $1 \mathrm{GeV}$ muon fluxes are far below expected electron/positron fluxes. In the given parameter

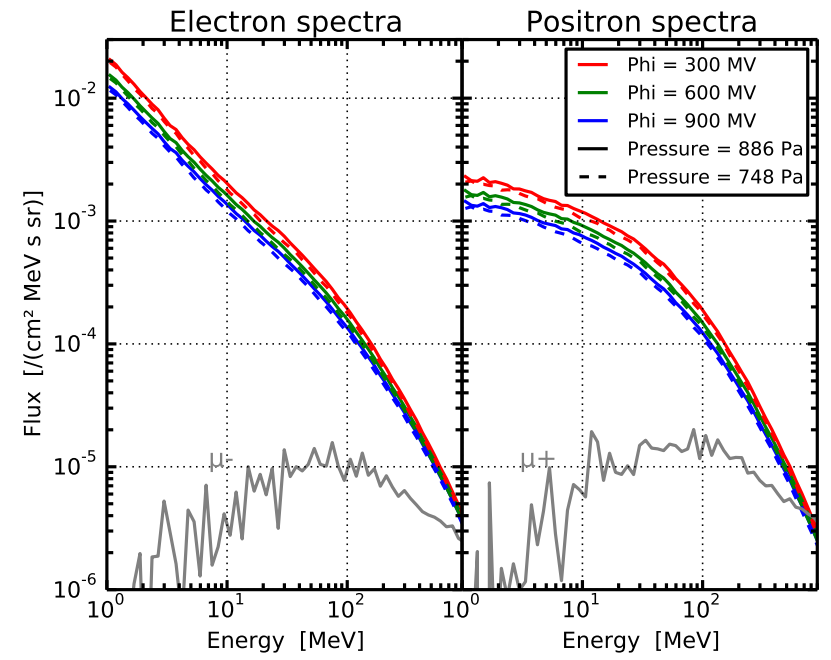

Figure 2. Electron and positron fluxes on the Martian surface calculated with Planetocosmics. The fluxes have been calculated for cosmic modulation values of 300 (red), 600 (green), and 900 (blue) $\mathrm{MV}$ and for pressure values of $886 \mathrm{~Pa}$ (solid) and $748 \mathrm{~Pa}$ (dashed). Muons and antimuons (gray) are shown for reference only (modulation potential $=600 \mathrm{MV}$, pressure $=748 \mathrm{~Pa}$ ).

range, electron/positron fluxes increase up to $\sim 90 \%$ comparing Martian summer, solar maximum with Martian winter, solar minimum.

\subsection{Modeling the RAD instrument}

Similar to other stopping charged particles, electrons and positrons can be identified by plotting the total deposited energy versus the energy deposited in A. However, they cannot be identified directly in a Goulding plot like the stopping charged particles in Ehresmann et al. (2014). To understand the behavior of electrons and positrons in RAD and to obtain selection criteria for electron/positron events, we model the electron/positron measurement process with the GEANT4 toolkit Agostinelli et al. (2003). The simulation is based on a detailed model of the instrument and includes effects such as electronic and optical noise in D, E, and F. This setup has been used in (Köhler et al., 2011, 2014; Ehresmann et al., 2014). As was done for stopping particles in Ehresmann et al. (2014), stopping electrons/positrons requires a coincidence between $\mathrm{A} 2 / \mathrm{A} 1$ and $\mathrm{B}$ and an anti-coincidence with $\mathrm{E}$ and $\mathrm{F}$.

The simulated instrument response for electrons, positrons and muons is shown in Fig. 3 (top). Particle spectra and relative intensities are used according to Planetocosmics simulation described above, with an atmospheric pressure of $886 \mathrm{~Pa}, \Phi=600 \mathrm{MV}$ (as shown in Fig. 2 as solid, green line).

Note that the measured muon events with a total deposited energy below $1 \mathrm{MeV}$ are mainly from high energy muons which create secondary particles in A, where only the sec- 


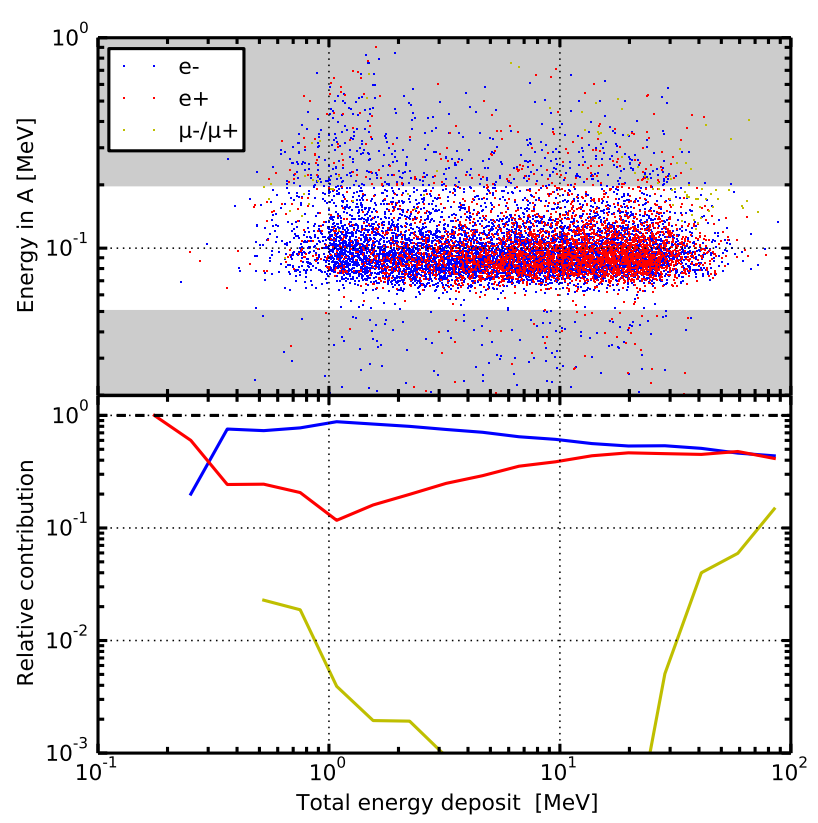

Figure 3. Top: simulated RAD stopping charge particle measurements for isotropic distributions of electrons (blue), positrons (red), muons and anti-muons (yellow) with spectra obtained from Planetocosmics simulations. The white area (between 50 and $200 \mathrm{keV}$ energy in A) marks the selection criterion for the RAD electron/positron measurement. Bottom: the expected energy dependent contribution of electrons, positrons and muons to the measurement.

ondary particles create further signals in B, C, and D. Measured muons with total deposited energy above $10 \mathrm{MeV}$ are at least partially caused by muons which stop in the B, C or $\mathrm{D}$ detector. Those stopping muons form an (barely visible) inclined line in Fig. 3 (top), ranging from ( $5 \mathrm{MeV}$ total energy deposit, $1 \mathrm{MeV}$ energy in A) to (100 MeV total energy deposit, $0.1 \mathrm{MeV}$ Energy in A). In a Goulding plot, such as presented in Ehresmann et al. (2014), this line of muons would be parallel to the $x$ axis. Possible contamination of the electron/positron measurement by other particle species has been investigated and found to be negligible. Using deposited energy in A and the total deposited energy, signals from electrons and positrons cannot be separated.

Based on the results shown in Fig. 3 (top), we require valid electron/positron events to have a deposited energy of 50$200 \mathrm{keV}$ in A. This selection criterion can be used to obtain a measurement of the total deposited energy of electrons and positrons. Figure 3 (bottom) shows the relative contribution of the different particle species to such a measurement.

Between 1 and $50 \mathrm{MeV}$ the intensity of the measured muons is far below the one of the electron/positrons and can be neglected. However, above $80 \mathrm{MeV}$ the relative intensity of the muons increases up to nearly $15 \%$ of the measurement. The quality of the obtained electron/positron selection criterion has been verified using electron, positron and muon spectra with different power laws and different relative in-

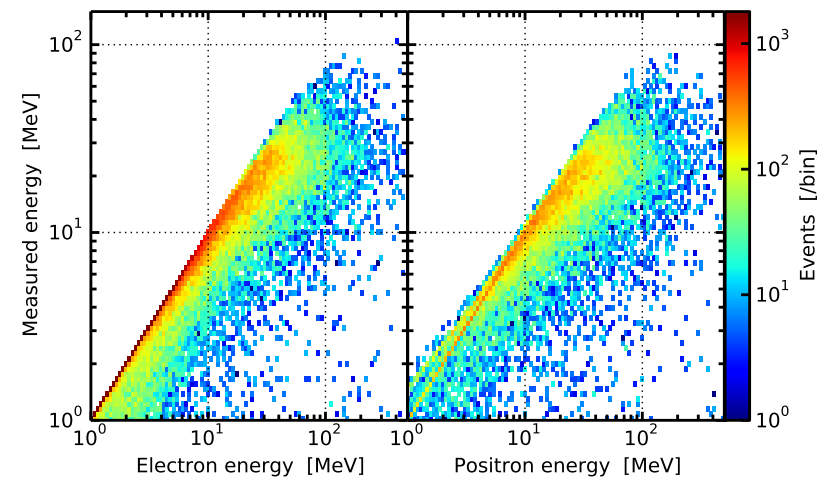

Figure 4. Detector response for stopping electrons (left) and positrons (right). The figure shows the measured energy versus the initial energy of the electron/positron for an isotropic distribution of particles and energy spectra obtained with Planetocosmics. The color denotes the number of measured particles per bin.

tensities. While the shape of the spectra does not influence the quality of the selection criterion, an increased intensity of the muon spectra will increase the relative contribution of the measured muons.

The corresponding modeled measurement of the total deposited energy is shown in Fig. 6 (black). Unlike the data in Figs. 3 and 4, the simulated measurement replicates the behavior of the RAD instrument as far as possible and includes instrumental effects like electronic and optical noise, as well as inefficiencies of the anti-coincidence (AC). Uncertainties caused by unknown AC threshold conditions are marked as gray area.

The wide scatter of signals in Fig. 3 already indicates that the total deposited energy of a stopping electron/positron does not necessarily correspond to its initial energy. To understand how the measured energy is related to the incident energy we use the GEANT4 simulation to obtain detector response, which maps incident versus measured energy for stopping electrons and positrons.

Figure 4 shows the detector response for electron and positrons with energies from 1-1000 MeV. For energies below $20 \mathrm{MeV}$ most of the deposited energies are almost equal to the initial energy; only a small fraction of the particles deposit energies significantly below their initial energy. In particular at higher energies, where particles should fully penetrate the instrument and therefore fail our event selection criteria, we find a significant share of events which appear as valid lower energy electrons/positrons. This happens, e.g., if an electron/positron creates secondary gamma rays, which leave the instruments without depositing their energy. Events where the measured energy does not reflect the particle energy and the similarity of electrons and positrons make it very difficult to obtain spectra from the measurements. Note that positrons can also deposit energies above their initial energies, due to gamma rays created through elec- 


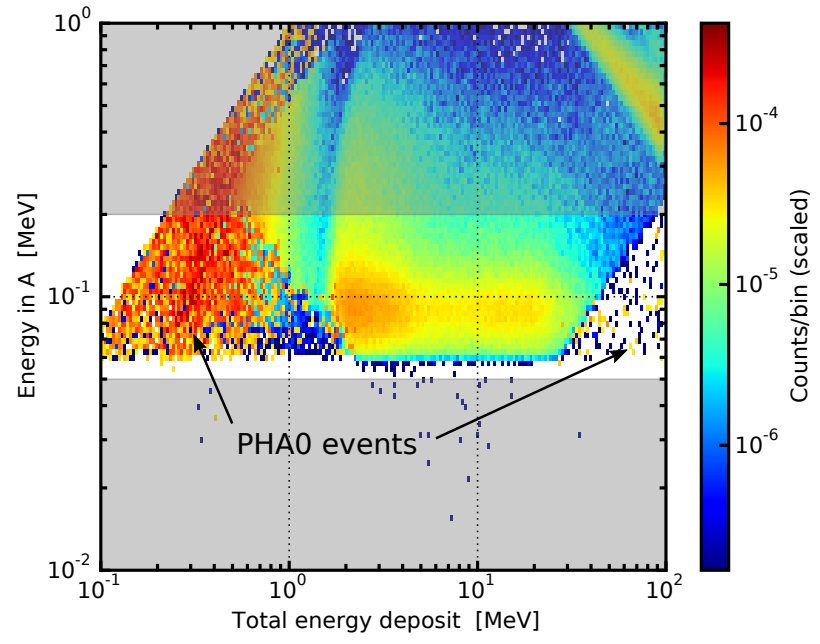

Figure 5. RAD stopping particle measurement for sol 400-500. As in Fig. 3, the gray area marks invalid electron/positron events. Single bins with high intensity are caused by PHA0 events, which have a large scaling factor. The corresponding measurement histogram is shown in Fig. 6.

tron positron annihilation. In principle, measuring a stopping positron in coincidence with a possible gamma from electron positron annihilation could be used to unambiguously detect positrons. However, such a measurement is currently not foreseen in the configuration of the RAD instrument.

\subsection{RAD electron/positron measurements}

Due to telemetry limitations, RAD stores and transmits only a subset of its pulse-height analyzed (PHA) data. RAD data are classified according to a priority scheme implemented in firmware, and for each priority only a subset of the PHA data is send back to Earth together with the corresponding scaling factors. To obtain the real number of PHA events, each event needs to be weighted with the corresponding scaling factor for its priority bin. A detailed explanation of this scheme is given in Hassler et al. (2012).

Most of the electron positron measurements are classified with priority 1 , which means that $80-98 \%$ of all available data is sent back to Earth. However, some electron/positron PHA records are classified with priority 0 , which means that only $\sim 0.1-0.25 \%$ of the available data is sent back to Earth. This results in scaling factors of $\sim 1-1.25$ for priority 1 events and $\sim 400-1000$ for priority 0 events.

Equivalent to the simulated data in Fig. 3, Fig. 5 shows the total deposited energy versus deposited energy in A from actual measurements during the period from sol 400 to $500^{1}$ (The time period corresponds to 20 September 2013 to 1 January 2014). During this period mean atmospheric pressure

\footnotetext{
${ }^{1} 1 \mathrm{sol}=1$ Mars day $\sim 1.03$ Earth day. The number of sols refers to number of sols since landing.
}

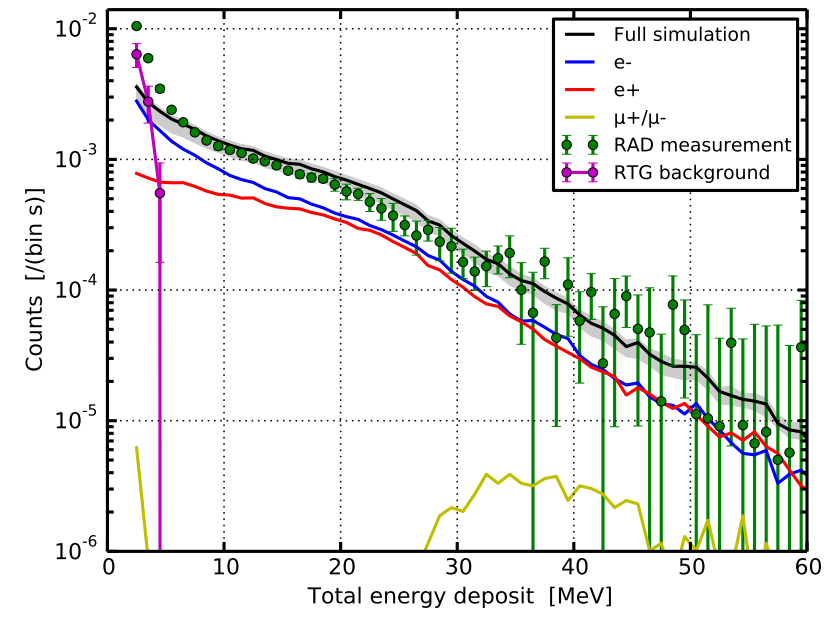

Figure 6. Simulated RAD measurement based on Planetocosmics calculation (compare Fig. 3) and RAD measurements for Sol 400500 (green) and RAD RTG background measurements (purple). Uncertainties in the simulated instrument behavior, caused by the not well known inefficiency of the AC, are marked as gray area. The individual contribution of simulated electrons, positrons and muons are shown in blue, red and yellow.

and mean value of $\Phi$ correspond to the values used in the Planetocosmics simulation discussed above. Priority 0 events are visible as isolated bins with high intensities, e.g., below $1 \mathrm{MeV}$ or above $20 \mathrm{MeV}$. The apparent gap at $1.5 \mathrm{MeV}$ is caused by the transition from electrons/positrons stopping in the $\mathrm{C}$ detector to those stopping in D. (There is a thin dead layer in between the two which causes the gap.) As expected from Planetocosmics predictions, there is no clear muon signal, however, stopping protons are visible at high energies, i.e., $300 \mathrm{keV}$ in $\mathrm{A}$ with total energies $>20 \mathrm{MeV}$. To obtain a clean measurement that is not influenced by instrumental effects, we select only events with total deposited energies above $2 \mathrm{MeV}$. One should note that total deposited energies above $20 \mathrm{MeV}$ are at least partially classified as priority 0 events. The fraction of priority 0 events gradually increases with energy. This results in large uncertainties at energies above $20 \mathrm{MeV}$ which cannot be assessed easily.

Figure 6 shows the histograms of the total deposited energies from both simulations and measurements which agree remarkably well. The measurement shows higher intensities at energies below $5 \mathrm{MeV}$, however, those are most likely caused by the Radioisotope thermoelectric generator (RTG). The purple line shows the RTG background measurement obtained during the "Hour of Power." (Before launch, with $\mathrm{Cu}$ riosity fully configured for the trip to Mars - including the installation of the RTG power source - RAD was turned on and acquired an hour's worth of data.) With the RTG background subtracted from the measurement, the simulation fully explains the measurement at low energies. One should note that the electron/positron flux produced by the RTG might depend 
on environmental conditions such as atmospheric pressure. Therefore the RTG electron/positron flux on Mars might deviate from the one measured on Earth with the spacecraft in the cruise configuration. For total deposited energies deposits above $20 \mathrm{MeV}$, the measurement contains priority 0 events, which have very low statistics and a large scaling factor $(\sim 800)$. This makes it very difficult to compare results of energies above $20 \mathrm{MeV}$. The gray area marks uncertainties of the $\mathrm{AC}$ threshold for $\mathrm{E}$ and $\mathrm{F}$ for the simulated measurement. The AC threshold for $\mathrm{E}$ is assumed to be $2400 \mathrm{keV}$ with an uncertainty of $\pm 200 \mathrm{keV}$. The AC threshold for $\mathrm{F}$ is assumed to be $400 \mathrm{keV}$ with an uncertainty of $\pm 100 \mathrm{keV}$. Varying the threshold for $\mathrm{E}$, one can increase or decrease the intensity at high energies $(>15 \mathrm{MeV})$; varying the threshold for $\mathrm{F}$, one can rescale the overall intensity. The gray area does not represent the statistical uncertainties but rather the systematic uncertainty.

\section{Discussion}

Figure 6 compares RAD electron/positron measurement for Sol 400-500 with Planetocosmics predictions. The pressure of $886 \mathrm{~Pa}$ and a modulation potential of $600 \mathrm{MV}$ were selected as average values for that period (compare Fig. 7). Pressure data were obtained from measurements of the REMS instrument on board the Curiosity rover (GómezElvira et al., 2012), and the modulation potential has been calculated from Oulu data ${ }^{2}$ using the method described in Usoskin et al. (2002).

The simulated and measured data in the energy range 4$20 \mathrm{MeV}$ have a very good agreement with each other. The systematic error, which accounts for uncertainties in the AC efficiency and represent absolute worst case values, results in a value of $\pm 20 \%$ in count rate. Figure 4 shows that the measurement is contaminated by high energy electrons and positrons, which means that the electron/positron measurements do not reflect the spectrum of incoming particles and also that a different combination of spectra might result in a similar measurement. That is, the measurement cannot be inverted to yield a unique solution for the incident electron and positron spectra. Figure 3 shows that electrons and positrons cannot be distinguished, i.e., in principle there are many possible ratios of electrons to positrons which would result in a similar measurement. Figure 3 also shows that there is a small muon background in the measurements, i.e., a significantly increased background of muons, combined with a decreased electron or positron intensity could also lead to similar results. Despite these caveats, the RAD measurement nonetheless provides verification of Planetocosmics predictions. Further measurements with more variable conditions (e.g., under more extreme modulation conditions) would clarify matters.

\footnotetext{
${ }^{2}$ The Oulu count rate data have been obtained from http:// cosmicrays.oulu.fi/ and the pressure effect has been corrected.
}

As an additional test we compared RAD electron/positron measurement for sol 600-700 with Planetocosmics predictions $(748 \mathrm{~Pa}, 600 \mathrm{MV})$, which yields a similarly good agreement as in Fig. 6. Comparing the results for sol 600-700 with the ones from sol 400-500, we notice that, for both simulation and measurement, with similar solar modulation levels, there is an increase of the electron/positron fluxes by $\sim 20 \%$ corresponding to the increase of the pressure. This indicates that the seasonal variations in the dose rate reported by Guo et al. (2015) are visible in the electron + positron measurement as well. However, instead of showing the shielding effect of the atmosphere, the electron + positron flux measurements reflect the secondary production of the atmosphere above the Pfotzer maximum.

\subsection{Seasonal variability of the electron/positron flux}

To investigate the temporal variability of the electron + positron fluxes we use the integrated count rate of the RAD electron/positron measurements. To avoid instrumental effects, and to minimize the RTG background, we use only deposited energies between 4 and $20 \mathrm{MeV}$.

In the previous section we demonstrated that the Planetocosmics predictions are in agreement with RAD measurements for two periods with different environmental conditions. For investigating the temporal variation of the electron + positron flux, we show both RAD electron + positron count rate and predicted Planetocosmics fluxes. In principle those Planetocosmics fluxes could be translated via the GEANT4 simulation setup into RAD electron counts. However, this is computationally expensive and would also add an additional element of uncertainty. Therefore, the seasonal variability of the RAD count rates are directly "compared" to the corresponding simulated Planetocosmics fluxes.

Figure 4 shows that the electron/positrons measurements between 4 and $20 \mathrm{MeV}$ not only contain electrons/positrons from $4-20 \mathrm{MeV}$, but also events from higher energies. The simulation shows that $\sim 25 \%$ of the deposited energies between 4 and $20 \mathrm{MeV}$ are caused by electrons + positrons with energies above $20 \mathrm{MeV}$. Nevertheless, we believe that the measurements are a good proxy for the integrated fluxes in the same energy range.

Because Planetocosmics simulations require a large amount of computation time, the simulations were conducted for a discrete set of pressures $(929,886,827,768,748,712$, $693 \mathrm{~Pa}$ ) and a discrete set of GCR spectra (modulation parameter $=500,510, \ldots, 800 \mathrm{MV}$ ).

In this work we do not attempt to model the dependence of particle fluxes on the solar modulation and pressure in detail other than to demonstrate the variability of the electron and positron flux. A detailed analysis of the influence of the solar modulation and pressure effects can be found in (Rafkin et al., 2014; Guo et al., 2015).

The solar modulation potential and the Martian pressure are shown in Fig. 7 (top, middle). Figure 7 (bottom) shows 


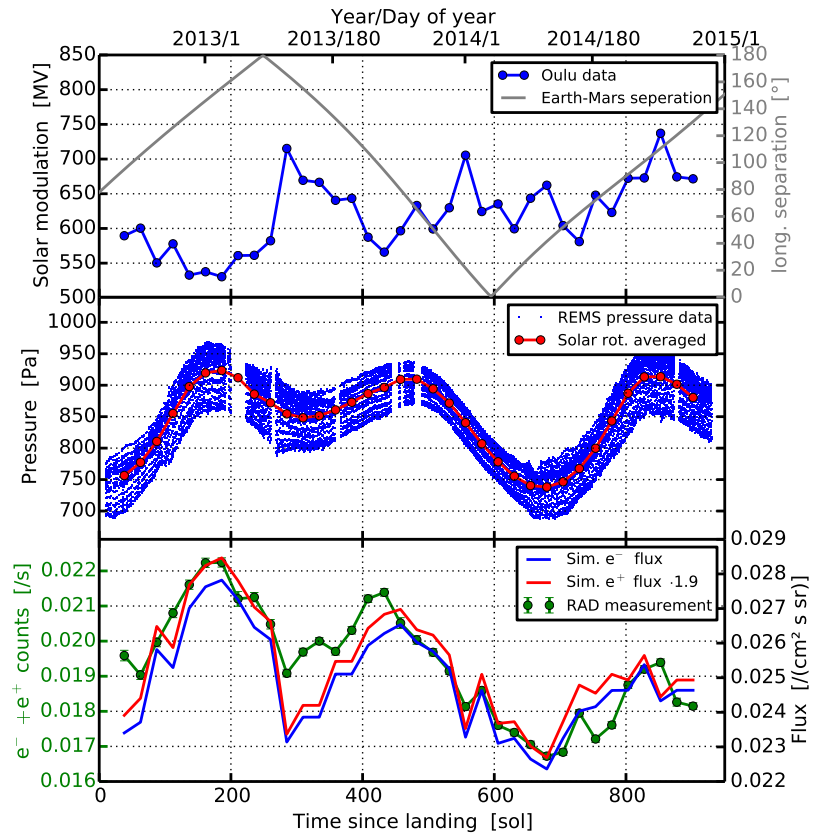

Figure 7. Top: solar modulation calculated from Oulu data (blue) and Earth-Mars longitudinal separation (gray). Middle: REMS pressure data. Bottom: RAD 4-20 MeV electron + positron count rate (green) and Planetocosmics prediction for 4-20 MeV electron and positron fluxes (blue, green). To minimize the influence of the solar rotation, the measurements and Planetocosmics predictions have been averaged over one solar rotation. The non-averaged REMS data show huge variations, which are caused by the diurnal variations.

the variation of RAD electron + positrons counts and the variation of simulated electron and positron fluxes. The error given for the RAD measurements represents the statistical error and the systematical error is not shown. To minimize the influence of the solar rotation on the solar modulation, e.g., smoothing out the difference of the solar modulation caused by the longitudinal spatial separation between Earth and Mars, all data have been averaged over one solar rotation. Since REMS pressure measurement are not performed continuously and the measurement cadence changes from sol to sol, as explained by Gómez-Elvira et al. (2014), a simple average of all measured values over a solar rotation period does not exactly match the actual pressure average. However, the errors caused by this and the calibration uncertainty of the pressure sensor ( $\sim 3 \mathrm{~Pa}$; Harri et al., 2014$)$ are small compared to the accuracy needed in this study and are therefore ignored.

Overall, the variations in Planetocosmics predictions agree quite well with the observed electron + positron fluxes. Around sol 250, the Planetocosmics predicts a strong decrease, which is less visible in the measured data. This difference is most likely caused by the large solar modulation parameter at Earth which does not necessarily represent the solar modulation at Mars, especially considering the large separation of Earth and Mars in heliospheric longitude during this period.

\subsection{Diurnal variability of the electron/positron flux}

The pressure-induced diurnal variations of the RAD measurements have been discussed by (Rafkin et al., 2014). As shown above, unlike for dose measurements, the electron + positron flux increases with pressure. To investigate the diurnal variability of the electron + positron fluxes we use the integrated count rate of the RAD electron + positron measurements. As above, we use only deposited energies between 4 and $20 \mathrm{MeV}$.

Following Rafkin et al. (2014) we compute correlation coefficient for linear regression (Pearson $R^{2}$ ) for the hourly perturbations of electron + positron counts and pressure. For sol 400-500 and for sol 600-700 we obtain a correlation coefficient of 85 and $90 \%$ respectively.

\subsection{SEP induced electron/positron flux}

Solar particle events have been observed by RAD on 12 April 2013 and 11 November, 6 January, and 1 September 2014. All four events are clearly visible as enhancements in the proton count rate and the dose rate measurement. However the integrated electron + positron flux shows no sign of enhancement during these events. Considering the available statistics and the resulting uncertainties, any possible enhancements of the electron/positron fluxes are below $5 \%$, which is well below the enhancements in dose rate of up to $90 \%$. This shows that typical SEP intensities or energies, or at least the intensities/energies of the four observed events, are too low to create significant enhancements of the electron flux on the Martian surface. Only protons with energies above $160 \mathrm{MeV}$ can penetrate the Martian atmosphere and reach the surface, i.e., most of the proton flux of SEP events stops in the upper atmosphere, and most of the electrons created there will not reach the surface. Those SEPs reaching the surface have lost most of their energy in the atmosphere and subsequent delta electron showers will have very low energies, well below the threshold of RAD.

\section{Conclusions}

We calculated the expected electron and positron fluxes with Planetocosmics. To compare these results with RAD measurements, we applied these spectra to a detailed model of the RAD instrument to simulate the measurement process with GEANT4. Simulation results are compared to measurements from sol 400-500 for which the average modulation potential and atmospheric pressure are assumed to be $600 \mathrm{MV}$ and $886 \mathrm{~Pa}$. Within the systematic errors $( \pm 20 \%)$, given by the somewhat uncertain efficiency of the anticoincidence system, the simulation can fully explain the measurement. However, since the measured signals from electrons and positrons 
cannot be distinguished in RAD, a different combination of electron and positron fluxes could lead to a similar result. Further uncertainties arise from the fact that the measured energy does not necessarily reflect the incident particles energy. This means that other spectra could, in principle, also lead to a similar result. Keeping all this in mind, and considering the demonstrated reliability of Planetocosmics, we believe that the RAD measurements provide a good verification of the Planetocosmics predictions.

Simulation and measurement were compared for a second measurement period (sol 600-700) for which the average modulation potential and atmospheric pressure are assumed to be $600 \mathrm{MV}$ and $748 \mathrm{~Pa}$. Compared to sol 400-500, both simulation and measurement show a $20 \%$ decrease in count rate, which can be attributed to decreased electron/positron generation above the Pfotzer maximum due to a decreased column depth. This is further investigated by comparing the temporal variability of the RAD electron + positron measurements with a Planetocosmics simulation for a large set of modulation potential and atmospheric pressure values. The trends of simulation and measurement show a good agreement for periods with low longitudinal separation between Earth and Mars and show some deviations for large angular separations.

Diurnal variations of the electron + positron flux, which has been reported for RAD dose rate as well as neutral particle count rates, could be observed and show a correlation coefficient of 85 and $90 \%$ for sol $400-500$ and sol 600-700 respectively.

No enhancements of the electron + positron fluxes during SEP events were observed in the data obtained to date, and the lack of such enhancements constrains them to be below $5 \%$ of the quiet-time fluxes.

Acknowledgements. RAD is supported by NASA (HEOMD) under JPL subcontract \#1273039 to Southwest Research Institute and in Germany by DLR and DLR's Space Administration grant numbers 50QM0501 and 50QM1201 to the Christian Albrechts University, Kiel. Part of this research was carried out at the Jet Propulsion Laboratory, California Institute of Technology, under a contract with the National Aeronautics and Space Administration. The data used in this paper are archived in the NASA Planetary Data System's Planetary Plasma Interactions Node at the University of California, Los Angeles. The archival volume includes the full binary raw data files, detailed descriptions of the structures therein, and higher-level data products in human-readable form. The PPI node is hosted at the following URL: http://ppi.pds.nasa.gov/.

The topical editor, M. Haberreiter, thanks two anonymous referees for help in evaluating this paper.

\section{References}

Adriani, O., Barbarino, G. C., Bazilevskaya, G. A., Bellotti, R., Boezio, M., Bogomolov, E. A., Bongi, M., Bonvicini, V.,
Borisov, S., Bottai, S., Bruno, A., Cafagna, F., Campana, D., Carbone, R., Carlson, P., Casolino, M., Castellini, G., Consiglio, L., De Pascale, M. P., De Santis, C., De Simone, N., Di Felice, V., Galper, A. M., Gillard, W., Grishantseva, L., Jerse, G., Karelin, A. V., Koldashov, S. V., Krutkov, S. Y., Kvashnin, A. N., Leonov, A., Malakhov, V., Malvezzi, V., Marcelli, L., Mayorov, A. G., Menn, W., Mikhailov, V. V., Mocchiutti, E., Monaco, A., Mori, N., Nikonov, N., Osteria, G., Palma, F., Papini, P., Pearce, M., Picozza, P., Pizzolotto, C., Ricci, M., Ricciarini, S. B., Rossetto, L., Sarkar, R., Simon, M., Sparvoli, R., Spillantini, P., Stochaj, S. J., Stockton, J. C., Stozhkov, Y. I., Vacchi, A., Vannuccini, E., Vasilyev, G., Voronov, S. A., Wu, J., Yurkin, Y. T., Zampa, G., Zampa, N., and Zverev, V. G.: Cosmic-Ray Electron Flux Measured by the PAMELA Experiment between 1 and $625 \mathrm{GeV}$, Phys. Rev. Lett., 106, 201101, doi:10.1103/PhysRevLett.106.201101, 2011.

Agostinelli, S., Allison, J., Amako, K., Apostolakis, J., Araujo, H., Arce, P., Asai, M., Axen, D., Banerjee, S., Barrand, G., Behner, F., Bellagamba, L., Boudreau, J., Broglia, L., Brunengo, A., Burkhardt, H., Chauvie, S., Chuma, J., Chytracek, R., Cooperman, G., Cosmo, G., Degtyarenko, P., Dell'Acqua, A., Depaola, G., Dietrich, D., Enami, R., Feliciello, A., Ferguson, C., Fesefeldt, H., Folger, G., Foppiano, F., Forti, A., Garelli, S., Giani, S., Giannitrapani, R., Gibin, D., Gómez Cadenas, J., González, I., Gracia Abril, G., Greeniaus, G., Greiner, W., Grichine, V., Grossheim, A., Guatelli, S., Gumplinger, P., Hamatsu, R., Hashimoto, K., Hasui, H., Heikkinen, A., Howard, A., Ivanchenko, V. N., Johnson, A., Jones, F., Kallenbach, J., Kanaya, N., Kawabata, M., Kawabata, Y., Kawaguti, M., Kelner, S., Kent, P., Kimura, A., Kodama, T., Kokoulin, R., Kossov, M., Kurashige, H., Lamanna, E., Lampén, T., Lara, V., Lefebure, V., Lei, F., Liendl, M., Lockman, W., Longo, F., Magni, S., Maire, M., Medernach, E., Minamimoto, K., Mora de Freitas, P., Morita, Y., Murakami, K., Nagamatu, M., Nartallo, R., Nieminen, P., Nishimura, T., Ohtsubo, K., Okamura, M., O’Neale, S., Oohata, Y., Paech, K., Perl, J., Pfeiffer, A., Pia, M., Ranjard, F., Rybin, A., Sadilov, S., Di Salvo, E., Santin, G., Sasaki, T., Savvas, N., Sawada, Y., Scherer, S., Sei, S., Sirotenko, V., Smith, D., Starkov, N., Stoecker, H., Sulkimo, J., Takahata, M., Tanaka, S., Tcherniaev, E., Safai Tehrani, E., Tropeano, M., Truscott, P., Uno, H., Urban, L., Urban, P., Verderi, M., Walkden, A., Wander, W., Weber, H., Wellisch, J., Wenaus, T., Williams, D., Wright, D., Yamada, T., Yoshida, H., and Zschiesche, D.: Geant4 - a simulation toolkit, Nucl. Instrum. Meth. A, 506, 250303, doi:10.1016/S0168-9002(03)01368-8, 2003.

Cleghorn, T. F., Saganti, P. B., Zeitlin, C. J., and Cucinotta, F. A.: Solar particle events observed at Mars: dosimetry measurements and model calculations, Adv. Space. Res., 33, 2215-2218, doi:10.1016/S0273-1177(03)00518-0, 2004.

Dartnell, L. R., Desorgher, L., Ward, J. M., and Coates, A. J.: Martian sub-surface ionising radiation: biosignatures and geology, Biogeosciences, 4, 545-558, doi:10.5194/bg-4-545-2007, 2007a.

Dartnell, L. R., Desorgher, L., Ward, J. M., and Coates, A. J.: Modelling the surface and subsurface Martian radiation environment: Implications for astrobiology, Geophys. Res. Lett., 34, L02207, doi:10.1029/2006GL027494, 2007b.

Desorgher, L., Flückiger, E., and Gurtner, M.: The PLANETOCOSMICS Geant4 application, 36th COSPAR Sci. Assem., 36, 2361, available at: http://adsabs.harvard.edu/abs/2006cosp...36. 2361D (last access: December 2015), 2006. 
Ehresmann, B., Burmeister, S., Wimmer-Schweingruber, R., and Reitz, G.: Influence of higher atmospheric pressure on the Martian radiation environment: Implications for possible habitability in the Noachian epoch, J. Geophys. Res., 116, doi:10.1029/2011JA016616, 2011.

Ehresmann, B., Zeitlin, C., Hassler, D. M., WimmerSchweingruber, R. F., Böhm, E., Böttcher, S., Brinza, D. E., Burmeister, S., Guo, J., Köhler, J., Martin, C., Posner, A., Rafkin, S. C. R., and Reitz, G.: Charged particle spectra obtained with the Mars Science Laboratory Radiation Assessment Detector (MSL/RAD) on the surface of Mars, J. Geophys. Res.-Planets, 119, 468-479, doi:10.1002/2013JE004547, 2014.

Gómez-Elvira, J., Armiens, C., Castañer, L., Domínguez, M., Genzer, M., Gómez, F., Haberle, R., Harri, A.-M., Jiménez, V., Kahanpää, H., Kowalski, L., Lepinette, A., Martín, J., MartínezFrías, J., McEwan, I., Mora, L., Moreno, J., Navarro, S., de Pablo, M. A., Peinado, V., Peña, A., Polkko, J., Ramos, M., Renno, N. O., Ricart, J., Richardson, M., Rodríguez-Manfredi, J., Romeral, J., Sebastián, E., Serrano, J., de la Torre Juárez, M., Torres, J., Torrero, F., Urquí, R., Vázquez, L., Velasco, T., Verdasca, J., Zorzano, M.-P., and Martín-Torres, J.: REMS: The Environmental Sensor Suite for the Mars Science Laboratory Rover, Space Sci. Rev., 170, 583-640, doi:10.1007/s11214-012-9921-1, 2012.

Gómez-Elvira, J., Armiens, C., Carrasco, I., Genzer, M., Gómez, F., Haberle, R., Hamilton, V. E., Harri, A.-M., Kahanpää, H., Kemppinen, O., Lepinette, A., Martín Soler, J., Martín-Torres, J., Martínez-Frías, J., Mischna, M., Mora, L., Navarro, S., Newman, C., de Pablo, M. A., Peinado, V., Polkko, J., Rafkin, S. C. R., Ramos, M., Rennó, N. O., Richardson, M., RodríguezManfredi, J. A., Romeral Planelló, J. J., Sebastián, E., de la Torre Juárez, M., Torres, J., Urquí, R., Vasavada, A. R., Verdasca, J., and Zorzano, M.-P.: Curiosity's rover environmental monitoring station: Overview of the first 100 sols, J. Geophys. Res.-Planets, 119, 1680-1688, 2014.

Guo, J., Zeitlin, C., Wimmer-Schweingruber, R. F., Rafkin, S., Hassler, D. M., Posner, A., Heber, B., Köhler, J., Ehresmann, B., Appel, J. K., Böhm, E., Böttcher, S., Burmeister, S., Brinza, D. E., Lohf, H., Martin, C., Kahanpää, H., and Reitz, G.: Modeling the Variations of Dose Rate Measured by RAD during the First MSL Martian Year: 2012-2014, Astrophys. J., 810, 24, doi:10.1088/0004-637X/810/1/24, 2015.

Harri, A.-M., Genzer, M., Kemppinen, O., Kahanpää, H., GomezElvira, J., Rodriguez-Manfredi, J. A., Haberle, R., Polkko, J., Schmidt, W., Savijärvi, H., Kauhanen, J., Atlaskin, E., Richardson, M., Siili, T., Paton, M., de la Torre Juarez, M., Newman, C., Rafkin, S., Lemmon, M. T., Mischna, M., Merikallio, S., Haukka, H., Martin-Torres, J., Zorzano, M.-P., Peinado, V., Urqui, R., Lapinette, A., Scodary, A., Mäkinen, T., Vazquez, L., and Rennó, N.: Pressure observations by the Curiosity rover: Initial results, J. Geophys. Res.-Planets, 119, 82-92, doi:10.1002/2013JE004423, 2014.

Hassler, D. M., Zeitlin, C., Wimmer-Schweingruber, R. F., Böttcher, S., Martin, C., Andrews, J., Böhm, E., Brinza, D. E., Bullock, M. A., Burmeister, S., Ehresmann, B., Epperly, M., Grinspoon, D., Köhler, J., Kortmann, O., Neal, K., Peterson, J., Posner, A., Rafkin, S. C. R., Seimetz, L., Smith, K. D., Tyler, Y., Weigle, G., Reitz, G., and Cucinotta, F. A.: The Radiation Assess- ment Detector (RAD) Investigation, Space Sci. Rev., 170, 60, doi:10.1007/s11214-012-9913-1, 2012.

Hassler, D. M., Zeitlin, C., Wimmer-Schweingruber, R. F., Ehresmann, B., Rafkin, S. C. R., Eigenbrode, J. L., Brinza, D. E., Weigle, G., Böttcher, S., Böhm, E., Burmeister, S., Guo, J., Köhler, J., Martin, C., Reitz, G., Cucinotta, F. A., Kim, M.-H., Grinspoon, D., Bullock, M. a., Posner, A., Gómez-Elvira, J., Vasavada, A. R., and Grotzinger, J. P.: Mars' surface radiation environment measured with the Mars Science Laboratory's Curiosity rover., Science, 343, 1244797, doi:10.1126/science.1244797, 2014.

Keating, A., Mohammadzadeh, A., Nieminen, P., Maia, D., Coutinho, S., Evans, H., Pimenta, M., Huot, J.-P., and Daly, E.: A model for Mars radiation environment characterization, IEEE Trans. Nucl. Sci., 52, 2287-2293, doi:10.1109/TNS.2005.860748, 2005.

Köhler, J., Ehresmann, B., Martin, C., Böhm, E., Kharytonov, A., Kortmann, O., Zeitlin, C., Hassler, D. M., and WimmerSchweingruber, R. F.: Inversion of neutron/gamma spectra from scintillator measurements, Nucl. Instrum. Meth. B, 269, 26412648, doi:10.1016/j.nimb.2011.07.021, 2011

Köhler, J., Zeitlin, C., Ehresmann, B., Wimmer-Schweingruber, R. F., Hassler, D. M., Reitz, G., Brinza, D. E., Weigle, G., Appel, J., Böttcher, S., Böhm, E., Burmeister, S., Guo, J., Martin, C., Posner, A., Rafkin, S. C. R., and Kortmann, O.: Measurements of the neutron spectrum on the Martian surface with MSL/RAD, J. Geophys. Res.-Planets, 119, 594-603, doi:10.1002/2013JE004539, 2014.

Lewis, S. R., Collins, M., Read, P. L., Forget, F., Hourdin, F., Fournier, R., Hourdin, C., Talagrand, O., and Huot, J.-P.: A climate database for Mars, J. Geophys. Res., 104, 24177, doi:10.1029/1999JE001024, 1999.

McKenna-Lawlor, S., Gonçalves, P., Keating, A., Morgado, B., Heynderickx, D., Nieminen, P., Santin, G., Truscott, P., Lei, F., Foing, B., and Balaz, J.: Characterization of the particle radiation environment at three potential landing sites on Mars using ESA's MEREM models, Icarus, 218, 723-734, doi:10.1016/j.icarus.2011.04.004, 2012.

O’Neill, P. M.: Badhwar-O'Neill 2010 Galactic Cosmic Ray Flux Model-Revised, IEEE Trans. Nucl. Sci., 57, 3148-3153, doi:10.1109/TNS.2010.2083688, 2010.

Potgieter, M. S. and Nndanganeni, R. R.: The solar modulation of electrons in the heliosphere, Astrophys. Space Sci., 345, 33-40, doi:10.1007/s10509-013-1365-x, 2013.

Rafkin, S. C. R., Zeitlin, C., Ehresmann, B., Hassler, D. M., Guo, J., Köhler, J., Wimmer-Schweingruber, R., Gomez-Elvira, J., Harri, A.-M., Kahanpää, H., Brinza, D. E., Weigle, G., Böttcher, S., Böhm, E., Burmeister, S., Martin, C., Reitz, G., Cucinotta, F. A., Kim, M.-H., Grinspoon, D., Bullock, M. A., and Posner, A.: Diurnal variations of energetic particle radiation at the surface of Mars as observed by the Mars Science Laboratory Radiation Assessment Detector, J. Geophys. Res.-Planets, 119, 1345-1358, doi:10.1002/2013JE004525, 2014.

Simpson, J. A.: Elemental and Isotopic Composition of the Galactic Cosmic Rays, Annu. Rev. Nucl. Part. S., 33, 323-382, doi:10.1146/annurev.ns.33.120183.001543, 1983.

Usoskin, I., Alanko, K., Mursula, K., and Kovaltsov, G.: Heliospheric modulation strength during the neutron monitor era, Sol Phys., 207, 389-399, doi:10.1023/A:1016266801300, 2002. 